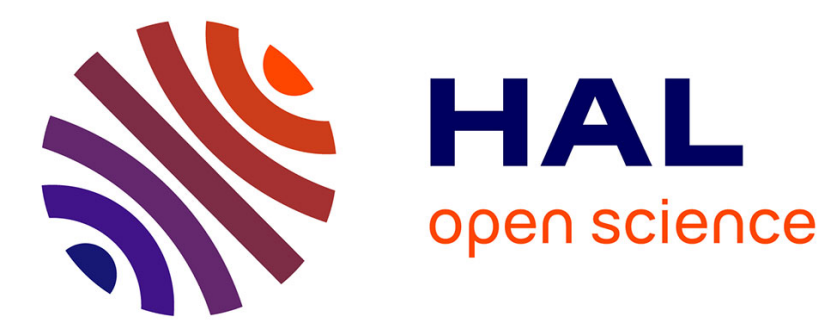

\title{
Les formes nominalisées du verbe basque
}

Didier Bottineau

\section{- To cite this version:}

Didier Bottineau. Les formes nominalisées du verbe basque. Faits de langues, 2008, 30, pp.15-39. halshs-00338501

\section{HAL Id: halshs-00338501 https://shs.hal.science/halshs-00338501}

Submitted on 13 Nov 2008

HAL is a multi-disciplinary open access archive for the deposit and dissemination of scientific research documents, whether they are published or not. The documents may come from teaching and research institutions in France or abroad, or from public or private research centers.
L'archive ouverte pluridisciplinaire HAL, est destinée au dépôt et à la diffusion de documents scientifiques de niveau recherche, publiés ou non, émanant des établissements d'enseignement et de recherche français ou étrangers, des laboratoires publics ou privés. 


\title{
Les formes nominalisées du verbe basque
}

\author{
Didier Bottineau*
}

\section{DE LA TYPOLOGIE DESCRIPTIVE A LA PROBLEMATIQUE}

Le basque est une langue non indo-européenne, agglutinante, ergative et à verbe final. Traiter de la question des formes nominalisées du verbe requiert son inscription dans l'économie générale de la grammaire de l'énoncé, qui se présente comme suit. A partir des données morphosyntaxiques (ordre des mots et agglutinations), on constate que le protocole énonciatif et interprétatif caractérisant l'énoncé basque prototypique - notion que l'on va préciser et relativiser - articule un domaine nominal (DN) et un domaine verbal (DV) réalisant respectivement une analyse et une synthèse :

$\begin{array}{lllll}\text { Udaletxeak aitari } & \text { eskutitza } & \text { bidali } & \text { dio. } \\ \text { mairie-DET-E } & \text { père-DET-D } & \text { lettre-DET } & \text { envoyé } & \mathrm{D}_{3} \mathrm{~A}_{3} \mathrm{E}_{3} \\ \text { «La mairie a envoyé une lettre à papa. » } & & \end{array}$

Le DN initial reçoit l'ensemble des syntagmes nominaux, actants et circonstants; le DV final reçoit le verbe à une forme simple ou composée (ici le participe accompli bidali et l'auxiliaire conjugué dio). La forme conjuguée finale, personnelle, est composée par accord multiple avec l'ensemble des actants appliqués (ici absolutif, ergatif et datif) : dio se décompose en $d^{\mathrm{A}}-i-o^{\mathrm{D}}-\emptyset^{\mathrm{E}}$, soit dans cet ordre «la-lui-elle ». Les éléments agglutinés à la voyelle actancielle $i$ sont des «pronoms liés » ${ }^{1}$ munis d'un rang personnel et d'une fonction; chacun renvoie individuellement à l'un des constituants nominaux de mêmes fonction et

*CNRS, MoDyCo, Université Paris 10 (Nanterre), didier.bottineau@ wanadoo.fr L'auteur remercie vivement Georges Rebuschi et Claude Hagège pour les multiples et riches commentaires et corrections proposés, et il est seul responsable de la version finale qui les intègre.

${ }^{1}$ Un couple personne / fonction donné comme «ergatif, première personne » est réalisé par un paradigme d'éléments formateurs de formes et placements variables et dont la sélection est déterminée par la co-occurrence d'autres actants et les repérages temporels et modaux : ikusi dut « je l'ai vu» $\left(\mathrm{E}_{1}=-t\right)$, ikusi nuen « je le vis » $\left(\mathrm{E}_{1}=n-\right)$. Pour simplifier on désigne ici par pronom liés ces classes de marqueurs morphosyntaxiquement variables et corrélatifs. Cette co-variation se présente très inégalement, mais non sans cohérence, selon les couples cas / personnes (Bottineau 2008). Le statut personnel et/ou modal des éléments formateurs est largement discuté (Trask 1981, Rebuschi 1999). 
rang personnel, à savoir eskutitza pour $d$, aitari pour $o$ et udaletxeak pour $\emptyset$. En son site propre, chaque pronom lié est une sélection paradigmatique à un rang personnel donné : dio « elle la lui », diot « je la lui », diozu « vous la lui », didazu «vous me la», diegu «nous la leur», dizkiguzu «vous nous les» etc. $\mathrm{Au}$ «présent » (en l'absence de marque de repérage temporel ou modal), la voyelle actancielle spécifie la nature de l'élément qui suit $(a+$ rien, $u+\mathrm{E}, i+\mathrm{D})$ et contribue à formater la matrice ${ }^{2}$. Ainsi, certains auxiliaires ${ }^{3}$, formés de la voyelle actancielle et de pronoms liés, sont-ils dépourvus de base lexicale, ce qui ne les empêche pas d'être utilisés comme «verbes $»^{4}$. Une évolution actuelle des dialectes du sud, dont est dérivée la norme euskara batua prescrite par l'académie Euskaltzaindia, en est peut-être l'indice, sinon la preuve : l'emploi croissant de verbes à racine lexicale en fonction d'auxiliaire (egon «être » pour l'uniactanciel, proche de l'espagnol estar; eduki «posséder», « avoir » pour le bi-actanciel) à côté des auxiliaires agglutinés de type $d a$ et $d u$.

\subsection{Conjugaison périphrastique}

Tous les verbes basques admettent une conjugaison périphrastique (CP) formée d'un participe et d'un auxiliaire dans cet ordre. On distingue trois participes d'un côté et quatre matrices actancielles auxiliées de l'autre : (i) le participe accompli (PA) ikusi «vu», le participe inaccompli (PI) ikusten «voyant » et le participe prospectif (PP) ikusiko « voir»; (ii) pour l'auxiliaire, on distingue quatre configurations valencielles construites sur trois cas, l'un obligatoire (absolutif A) et deux facultatifs (ergatif E, datif D) : A $d a$ «il est», EA $d u t$ « je le », DA zait « ça me », EDA diot « je le lui ». Par contraste avec les langues romanes, la variation aspectuelle du participe et la variation actancielle de l'auxiliaire fonctionnent indépendamment l'une de l'autre : tous les aspects du participes sont compatibles avec toutes les valences de l'auxiliaire. En faisant varier l'aspect, on distingue bidali dio «elle la lui a envoyée » (PA), bidalitzen dio « elle le lui envoie» $(\mathrm{PI})$ et bidaliko dio « elle la lui enverra » (PP); et en faisant varier la valence on obtiendra bidali dio « elle la lui a envoyée » (EDA),

\footnotetext{
${ }^{2}$ A y est nécessairement représenté, $\mathrm{E}$ et $\mathrm{D}$ sont optionnels, ce qui donne 4 matrices : A, $\mathrm{AD}, \mathrm{AE}, \mathrm{ADE}$, plus les « conjugaisons allocutives»; pour certains verbes «impersonnels» un voire plusieurs éléments (A et/ou $E$ ) ne reprennent pas un SN restituable (Bottineau \& Roulland 2008). Le dispositif se conserve aux temps autres que le présent mais sans -a\# (toujours final, incompatible avec un suffixe de temps ou de mode) et préfixation de E relativement à - $u$ - .

${ }_{3}^{3}$ Par contre les auxiliaires constitutifs du potentiel et du subjonctif présentent une racine lexicale -za- à laquelle s'agglutinent les pronoms liés : ikus dezaket «je peux le voir», ikus dezadan «que je le voie ».

${ }^{4}$ Nik emaztea dut 'moi-E femme-A A3- $u$-E1' «moi j'ai une femme» («moi je suis marié ») : la notion avoir peut s'exprimer soit par un «auxiliaire » dépourvu de base lexicale, soit par un verbe muni d'une telle base : daukat $\mathrm{A}_{3}-\ll$ posséder »- $\mathrm{E}_{1}$ «je possède ».
} 
bidali $d u$ « elle l'a envoyée » (EA) et bidali $d a$ « elle est envoyée / partie $»^{5}$. On distingue ainsi trois formes non conjuguées ou impersonnelles à signifié aspectuel, superficiellement comparables aux formes impersonnelles des verbes romans (infinitif, participes «présent» et «passé »). Mais elles s'en distinguent par plusieurs traits : (i) la modularité des alternances aspectuelles (participes) et actancielles (auxiliaires) empêche la formation de périphrases solidarisées par des contraintes morphosyntaxiques liées à des appariements de valence et d'aspect (tels que do + base verbale, be $+\mathrm{V}$-ing et have + participe passé en anglais) et permet l'occultation d'arguments dans des structures telles que le medio-passif (un agent indéfini et un patient défini, auxiliaire uniactanciel) : Noiz bidaltzen da ALTa? «Quand envoie-t-on l'ITA ${ }^{6}$ ?» («Quand ce document s'envoie-t-il ? »). (ii) Parmi les trois participes basques, la forme lemmatique est le PA (homologue aspectuel du participe passé roman) et non le PP (homologue de l'infinitif) qui en est dérivé par une postposition génitive : ikusi, ikusiko. Le $\mathrm{PA}$ sert notamment à la nomination du procès accompagnant les verbes modaux : ikusi nahi $d u$ « je veux le voir».

\subsection{Conjugaison synthétique}

D'autre part, le basque conserve pour un nombre limité de verbes (douze verbes courants, vingt-quatre verbes en tout selon la liste prescriptive de l'académie de la langue basque Euskaltzaindia) une conjugaison synthétique (CS) ; elle consiste à agglutiner les pronoms liés au radical même du verbe lexical, lequel ne reçoit aucune marque aspectuelle: daramakiot «je le lui apporte »(EDA), daukat « je l'ai » (EA), nator « je viens » $(\mathrm{A})$, natorkio « je viens à lui »(DA). L'absence de marque aspectuelle réserve ces formes à des valeurs ponctuelles ou singulatives, en particulier dans les emplois impératifs : emaidazu «donnez-le moi ». En CP, les auxiliaires correspondants (de même actance au même temps) sont diot (EDA), dut (EA), da (A) et zait (DA), puis iezaidazu (EDA impératif). Les quatre premiers s'associent à n'importe lequel des trois participes aspectualisés. En somme, les formes à caractère nominal ou adjectival, les participes, s'obtiennent en délocalisant l'ensemble de

\footnotetext{
5 Irteerako mezu bat bidali da «Un message sortant a été envoyé » («s'est envoyé », « est parti » - cf. anglais Did it send this time? «Il est parti cette fois ?») : procès accompli. Avec suffixe - a résultatif : Ez dira [nik bidali]ak. Litt. 'Ce ne sont pas des [envoyés de/par moi]' : "ils n'ont pas été envoyés par moi » (Jérémie 29, 8; TOB : «Je ne les ai pas envoyés »), structure généralement traduite par un passif. Le parfait est obtenu par l'ajout de l'auxiliaire izan: Zure mezua ongi bidali izan da "Votre message a bien été envoyé ». On peut cumuler le parfait (izan) et le résultatif $(-a)$, ce dernier remplaçant alors ongi «bien» (la confirmation, au lieu d'être posée lexicalement par l'adverbe, l'est gramamticalement par le résultatif) : Zure mezua bidalia izan da «Votre message a bien été envoyé ».

${ }^{6}$ Document administratif de la Sécurité Sociale espagnole.
} 
l'agglutination actancielle pronominale, construite en un tout autonome, «l'auxiliaire », totalement dépourvu de racine lexicale?

\subsection{Structure de l'assertion indépendante}

Un énoncé basque est «canonique », d'ordre non marqué et indépendant du contexte, lorsque tous les arguments nominaux (actants et circonstants) sont explicites et précèdent le groupe verbal final (CS ou CP). Les variations concernent diverses implicitations et extrapositions (par focalisation, thématisation, négation, passivation etc.). Ce protocole énonciatif rappelle celui décrit par Culioli dans des énoncés français oraux du type, «Mon frère, sa moto, le guidon, le chrome, il est parti $»^{8}:$ «la mairie-la, papa-à, lettre-la, envoyer, lalui-elle ». Le DN analytique décompose la scène en une série de participants à rang personnel et à fonctions dont un noyau obligatoire A et deux satellites adnominaux optionnels $\mathrm{D}$ et $\mathrm{E}$, formant une matrice subjectale multiargumentale à centre nominal absolutif, un sujet complexe ${ }^{9}$. Pour sa part, le DV synthétique incorpore ce sujet complexe (avec ses couples rang / cas) soit au

7 Les grammaires traditionnelles ramènent la valence intransitive à un auxiliaire izan « être » et les valences multiples à un auxiliaire ukan « avoir ». L'observation des données morphologiques et diachroniques rendent cette position difficilement tenable vu l'absence des bases - $z a$ - et $-u k a$ - des conjugaisons auxiliées (Bottineau 2008c) et la présence de $u k a$ - dans la conjugaison du verbe eduki « posséder » (cf. daukat « je le possède »).

8 Ressemblance en fait superficielle : outre la différence de registre, il s'agit en français d'un chaîne de thèmes enchâssés et non d'arguments hiérarchisés, comme en témoigne l'ordre libre moi, le gateau / le gateau, moi, je le mange. Cela étant, le français permet, par scalarisation des thèmes, un parcours analytique discursif des participants appelés à être repris comme arguments cliticisés autour du verbe, démarche énonciative proche proche de la phase analytique basque.

9 Le statut du sujet en basque, voire son existence même, sont largement débattus (traditions multifactorielles d'identification du sujet par faisceau de traits: Anderson 1976, Rotaetxe 1978; Rebuschi 1978, 1984 : ces traits subjectaux sont pour le basque distribués sur divers cas, rendant la notion de sujet inutile; Roulland 2004 : l'accord multiple invalide la notion de sujet en basque ; Coyos 1999: pas de sujet, mais des actualisateurs verbaux généralisé (absolutif) et non généralisé (ergatif) (approche fonctionnaliste unifactorielle). Bottineau 2007 : notre analyse, peut-être pas incompatible avec celle de Coyos, traite l'absolutif nominal comme un déterminant obligatoire du verbe (instancié ou non - il y a des structures «impersonnelles » diverses) modifiable par des adnominaux (ergatif et datif); la détermination verbale enregistre ensemble les traces morphologique de l'absolutif et celle des adnominaux qui le prémodifient en amont de l'accord verbal, le faisant paraître «multiple». Le «multisujet» est une sorte de «stemma nominal » avec A pour noyau obligatoire et $\mathrm{E}$ et $\mathrm{D}$ pour satellites optionnels (D étant différemment incorporé selon les dialectes). La prémodification réciproque des nominaux détermine (i) la souplesse du rôle sémantique associable à l'absolutif (agent isolé, patient, source d'affect, trajecteur selon les combinaisons de cas et (ii) la variation de chaque élément formateur verbal de chacun en fonction des co-occurrences des autres : un absolutif peut être marqué différemment selon qu'il est prémodifié par un ergatif ou non ; (Bottineau 2008c) la marque des parties du réseau varie en fonction de l'état général du tout (co-occurrence d'arguments et de repérages temporels et modaux). 
verbe $(\mathrm{CS})$, soit à l'auxiliaire de la $\mathrm{CP}: \mathrm{E}_{1} \mathrm{D}_{3} \mathrm{~A}_{3}=$ diot au présent. Cette incorporation est combinable à des repérages de temps ${ }^{10}$, de modalité ${ }^{11}$ et d'allocutivité ${ }^{12}$, cumulables ${ }^{13}$. Dans cette agglutination, la forme et position des pronoms liés varie au gré des configurations actancielles et des paramétrages temporels, modaux et allocutifs, alors que les formes mêmes de ces paramétrages sont fixes et en forme $(-n,-k e, k a / n a)$, et en position pour les deux premières : le profilage du multisujet incorporé est contraint par le paramétrage prédicationnel verbal.

Tout ceci fait de l'assertion un scenario cognitif ${ }^{14}$ articulant une séquence analyse / synthèse : le DN compose le dramatis personae par couplage nominal actants / acteurs en amont, et en aval, le DV exécute l'intrigue par agglutination verbale en confrontant le multisujet aux paramètres prédicationnels ${ }^{15}$. La CS concentre autour du radical du verbe lexical la fusion des actants nominaux, alors que dans la CP ce radical, muni de suffixes aspectuels participiaux, conclut le DN analytique en spécifiant notionnellement le procès et en quantifiant son déroulement; la synthèse prédicationnelle est alors concentrée dans l'auxiliaire final. Ceci confère aux formes participiales une fonction dans l'économie générale du système absolument distincte de celle que l'on pourrait assigner à leurs homologues romans.

10 Temps : $\varnothing$ vs - $n$ (passé), soit diot vs nion; dans cette configuration, - $t$ final de $\operatorname{diot}$ (E1) « je la lui » est remplacé par $n$ - initial de nion, $-n$ final étant la marque de passé.

11 Modalité : $\varnothing$ vs -ke (hypothèse), soit diot vs nioke (avec maintes fluctuations allomorphiques et syntaxiques dans la composition).

12 Parmi les éléments formateurs, on peut ajouter un allocutif masculin $\mathrm{ka}$ ou féminin $\mathrm{na}$ ( $2^{\mathrm{e}}$ personne singulière) ne correspondant à aucun argument nominal du procès mais destinant la prédication à un allocutaire spécifiquement ciblé : diot (déclaratif) $>$ zioat 1 zionat (allocutif), nioke > niokek / nioken, etc. : «je te dis que + le-lui-je + temps / modalité ».

13 Par ex. diot porté à l'hypothétique $(-k e)$ passé $(-n)$ et allocutif féminin $(n a)$ se formule niokenan.

14 «scenario cognitif» (Bottineau 2008a et b), «parcours prédicationnel» (Bottineau 2005): l'enchaînement typiquement suivi dans une langue donnée pour former une « idée » par l'acte de parole en exécutant les étapes réglées par la syntaxe propre à un type linguistique et les opérations propres à ses unités morphologiques (récupération de prototypes lexicaux, interconnexions par les marqueurs grammaticaux). Ce scenario est celui de l'interprétation, tant par l'allocutaire (parole vocale effective) que pour le locuteur lui-même (discours intérieur non vocalisé, vecteur de la constitution de la pensée réflexive) : la parole un enchaînement vocal suscitant une cascade d'événements cognitifs distribués à autrui et à soi (par proprioception ou simulation intérieure); elle permet l'émergence à la conscience d'une représentation non nécessairement intentionnelle ou planifiée. En acquisition, l'enfant l'incorpore par l'expérience orale et la segmentation graduée du discours de l'adulte qu'il finit par restituer énonciativement.

${ }^{15} \mathrm{La}$ phase analytique est toujours représentée par au moins un actant ou circonstant. Si tous sont implicites en contexte, elle est remplacée par le préfixe verbal $b a$-, forme réduite de bai «oui », servant d'appui obligatoire pour un verbe précédé de rien: *dakit > Badakit « je sais » / « je le sais », vs Nik dakit « moi je sais ». Hormis l'impératif, le verbe personnel n'est normalement pas initial (à l'exception de certains dialectes basques de France). 


\subsection{Syntagmes nominaux (SN) et propositions subordonnées (PS)}

On regroupe ici le $\mathrm{SN}$ et la PS parce qu'à des niveaux de développement fractal distincts (Robert 2003) ils instancient exactement la même structure ${ }^{16}$ : la forme schématique des marqueurs est la même quelle que soit la nature du syntagme lexical auquel elle s'applique. Le SN basque se décompose en deux segments successifs, l'un lexical / notionnel et l'autre grammatical / fonctionnel : [etxe][a] «[maison][la]».

Le premier segment peut être instancié (i) par un groupe nominal de la forme [((ADV)) N (ADJ)], par exemple [((oso)) emakume (polit)] «((tout)) femme (beau) »> «très belle femme »; (ii) par une proposition terminée par une forme personnelle du verbe en CS ou CP (section 3.) ; et (iii) par une proposition terminée par une forme impersonnelle du verbe, soit le nom verbal, soit le participe accompli (sections 4. et 5.).

Le second segment, grammatical / fonctionnel, reçoit les postpositions casuelles ${ }^{17}$ spécifiant les «cas » nominaux, la détermination et le nombre du référent. On distingue les cas actanciels exclusifs (incorporables au sujet verbal: absolutif, ergatif, datif) des cas inactanciels inclusifs. Les premiers (absolutif, ergatif, datif) sont mutuellement exclusifs et non surcomposables (à la manière des cas latins) et actanciels (pris en compte dans la formation du multisujet

16 Stéphane Robert reconnaît dans les marques grammaticales les propriétés des objets fractals de Benoît Mandelbrot (1975). La démarche théorique demeure à la fois confidentielle et controversée, soulevant au moins deux questions (selon nous) : (i) la pertinence du transfert et (ii) la dimension cognitive. Soit concrètement l'exemple de -ko: le participe «futur» (bidaliko «envoyer») et le génitif locatif etxeko «de la maison » peuvent-ils être perçus par le linguistes comme des objets fractals munis de caractéristiques communes (la forme schématique de Culioli selon Robert) ajustables par confrontation aux propriétés d'échelles différenciatrices des environnements syntaxiques d'insertion? Et si oui, (ii) faut-il postuler une dynamique cognitive permettant le couplage de l'invariant schématique d'une forme à la variation des syntagmes ciblés ? L'enjeu du débat est qu'il permet de relativiser et conserver la notion d'invariance en la rendant compatible avec celles de reprofilage (Cadiot \& Visetti 2001) et éventuellement de constructions (Goldberg 1995) selon une logique de couplage. Toujours est-il qu'on trouve bien deux formes du participe prospectif -ko / -en correspondant aux deux génétifs nominaux (cf. note 14 infra), de distributions dialectalement variables.

17 Terminologie : les postfixes grammaticaux sont envisagés comme objets fractaux munis d'un invariant schématique et d'une variation combinatoire. Au premier niveau il conviendrait de les nommer relateurs sans plus. Au second niveau il faudrait en principe bannir la terminologie classique (postpositions, cas) au sens où ces marques ne relèvent pas de la flexion lexicale mais bien du chaînage des marques de relation liant un syntagme lexical (à gauche) à un autre (à droite). Par souci d'économie et de lisibilité on n'intègre pas à cette étude de révision terminologique massive et on se contente de sélectionner les appellations en fonction des propriétés des marqueurs dans les environnements morphosyntaxiques où ils sont envisagés ( « cas », «(sur)déclinaison », etc.), et on parle de «postpositions» plutôt que «postfixes» ou «suffixes» en raison de la portée syntagmatique - non lexicale - des relateurs. 
contrôlant l'accord verbal) ${ }^{18}$; les seconds sont inactanciels (pas d'accord) et cumulables: INSTR + GL -z-ko (aurrezko «en or»), ADL + GL -ra-ko (Bilborako bidea «la route d'à Bilbao», etc. Ce complexe grammatical se suffixe au dernier constituant lexical du segment notionnel du SN, portant en facteur commun sur l'ensemble syntagmatique qui précède : etxeko atea «la porte de la maison», [etxe gorri]ko atea « la porte de la maison blanche ». Si le segment notionnel, propositionnel, se termine par une forme impersonnelle ou personnelle du verbe, celle-ci reçoit en suffixe le complexe grammatical casuel comme le ferait un nom ou un adjectif. -ko présente une diversité spectaculaire d'autres appariements, nominaux et verbaux : (i) Adv. : atzoko egunkaria « le journal d'hier» ; (ii) Adv. de manière en -la: holako lana « ce genre de travail »; (iii) Locution adverbiale: hitzez hitzeko itzultzea «traduction mot à mot»; (iv) NP à un cas spatial: mendietako haitzulak "les grottes de (dans) la montagne" (mendietan inessif pluriel $+k o)^{19}$; (v) NP comitatif (-ekin-): gureki(la)ko umeak "les enfants de (que) avec nous"; vi) NP instrumental (-z-) : urrezko « en or »; vii) avec postposition : lotsagabeko emakume bat « une femme de sans honte »; (viii) NP indéterminé ou en -ka (adverbe d'itérativité) : bai-ala-ezkako galderak «yes-no questions» (bai « oui », ez «non»); (ix) participe adverbial en -ta (résultatif) or -rik (partitif) : amak ikusitako sagua «la souris vue par maman »; x) subordonnée circonstancielle à verbe fini : izarra agertu zitzaieneko garaian «à l'époque où les étoiles leurs sont apparues"; (xi) une complétive en -la (discours rapporté) : hil dutelako kontua «le rapport selon lequel ils l'ont tué »; (xii) nom verbal en -tze : sagua ez hiltzeko esan diot « je lui ai dit de ne pas tuer la souris »; (xiii) participe prospectif : hilgo $d u$ « il la tuera ». Le seul cas où le segment fonctionnel est antéposé au nom en tout ou en partie est celui où la référence ou le nombre sont mis en question : zein etxe «quelle(s) maison(s) », zenbat etxe «combien de maisons »: sont antéposés les marqueurs de paramètres que le locuteur ne fixe pas lui-même (et éventuellement délègue à l'allocutaire) ; la présupposition peut répartir les marqueurs ${ }^{20}$.

\subsection{Système d'interprétation}

Il existe trois formes participiales fondamentales obtenues par délocalisation de l'agglutination du sujet personnel complexe vers un auxiliaire formel final autonome, et suffixation de marques aspectuelles; ceci concerne le verbe en tant qu'unité lexicale. En l'absence de négation, le verbe en forme personnelle occupe canoniquement la place finale de l'indépendante. Or celle-ci peut être enchâssée dans une proposition supérieure en saturant le segment notionnel d'un GN. Son ultime constituant, le verbe conjugué, recevra les marques de déclinaison

18 Pour le souletin, le datif, non pris en compte dans l'accord verbal, doit être exclu des cas actanciels.

19 Selon Hualde et Urbina 2003, 146-9.

20 Zein gizonak esan du hori? «quel homme a dit ça?» ('quel l'homme', ergatif déterminé - il est présupposé qu'il y en a bien un). 
nominale comme le ferait un nom ou un adjectif. De même, le verbe impersonnel final d'une subordonnée apparaîtra comme « déclinable ».

Dans ce système, il convient de se demander si les formes nominales observées sont bien celles du verbe en tant qu'unité lexicale de langue, ou celles de la proposition en tant que constituant syntagmatique du discours, qu'il termine par contrainte typologique et cognitive ${ }^{21}$. La notion même de «forme nominale » pourrait s'avérer un contresens partiel pour certaines formes du verbe basque.

\section{LES TROIS BASES DU VERBE BASQUE}

La CP disjoint le verbe en auxiliaire (multisujet et prédication) et participe impersonnel par déflexité. Cette soustraction de l'agglutination prédicationnelle livre une racine lexicale non libre, la base zéro (Bottineau 2006), à partir de laquelle plusieurs niveaux de construction de formes impersonnelles à caractère participial, nominal ou adjectival sont possibles :

- Base 0, « racine » dans la terminologie traditionnelle. - Pour un verbe comme $i k u s i$ « voir », le noyau sémantique est une racine non libre, ici -kus-, que l'on a nommée B0 et que l'on ne trouve jamais réalisée sous cette forme : elle doit être affixée, soit par le complexe arguments / paramètres (conjugaison synthétique), soit par des marques de repérage aspectuel.

- Base 1, «radical» dans la tradition. - Cette forme n'est pas toujours distincte de la racine (en raisons de contraintes morpho-phonologique essentiellement). Lorsqu'elle l'est, elle s'obtient par préfixation d'un élément vocalique ou consonantique : pour ikusi «voir», racine $=-k u s-$, radical $=i k u s$. Ce préfixe oblitère le couplage arguments / paramètres et annonce une forme verbale apersonnelle, atemporelle, amodale et «inallocutive », interdisant en bloc toutes les configurations actancielles et prédicationnelles. Cette forme est virtuelle et aoristique au sens où tous les types de repérages mentionnés sont occultés : elle est compatible avec l'auxiliaire au subjonctif pour l'expression d'un procès envisagé dont l'actualisation personnelle et prédicationnelle n'est pas établie, ikus dezadan "pour qu'il le voie»; avec l'auxiliaire impératif, visant à l'actualisation du procès ikus ezazu «voyez-(le)»; avec l'auxiliaire potentiel pour l'expression du possible, ikus nazake «il peut me voir ». Par cette virtualité

21 Bottineau 2007: la morphosyntaxe phrastique caractérisant typologiquement une langue donnée est révélatrice de la procédure énonciative suivie dans cette langue pour faire construire le sens par l'interprétant, qu'il s'agisse d'un allocutaire (acte de communication vocale ou graphique) ou du locuteur lui-même (conducteur de l'émergence de ses idées conscientes par l'acte de langage intérieur, la pensée discursive) ; la phrase est un algorithme idéo-génétique sous contrôle individuel et à effets socialement distribués et variant selon les contextes psychologiques des réceptions (avec leurs historicités propres). La procédure basque, de type analyse / synthèse, assigne au verbe une fonction de regroupement manifestée par sa morphologie (incorporation d'éléments formateurs) comme par sa syntaxe (propension à occuper la dernière place de la proposition qu'il verrouille). La contrainte typologique (qui en acquisition s'impose à l'enfant par les modèles parentaux) est constitutive d'une procédure cognitive (l'acte de formation du sens, bien distincte en basque par comparaison au français ou au breton). 
même, la B1 est incomplète, elle ne peut jamais occuper la dernière position d'une indépendante ou d'une subordonnée, elle ne peut pas sans auxiliaire instancier le segment notionnel d'un SN, et elle ne peut pas recevoir de marque de cas, détermination ou nombre : *ikusa, *ikusak, etc. En un mot, cette forme impersonnelle n'est ni nominale, ni adjectivale; en basque, l'impersonnalité n'entraîne pas mécaniquement la translation verbe-nom (au sens tesniérien). Enfin, la B1 peut recevoir un suffixe -tze (avec divers allomorphes -te, -zte) formateur du nom verbal (NV) ikuste «fait / action de voir» (suivi de postpositions casuelles) et du participe inaccompli (PI) ikusten (cf. 1.1. supra), forme archaïque de l'inessif indéfini de l'infinitif nominal employé comme gérondif verbal selon Lafitte $(1979,215-6)$.

- Base 2, «participe passé » ou «participe accompli » (PA) dans la tradition. Lorsqu'elle est distincte, cette forme s'obtient par ajout d'un morphème final à la B1 : ikus > ikusi. A la forme virtuelle ikus (réalisation nulle de l'événement) s'oppose la forme actuelle ikusi (réalisation intégrale du commencement à la fin, avec télicité et perfectivation). Le contraste concerne à la fois le mode et l'aspect : ikus, irréel, est aspectuellement nul (aucune saisie, même inchoative) : ikusi, réalisé, est aspectuellement complet (terminatif). Les actualisations modales et aspectuelles, traitées par des systèmes distincts dans les langues romanes, sont indissociablement liées en basque. C'est pour cette raison que la forme lemmatique du verbe actualisé est la B2 ikusi d'aspect perfectif ou terminatif, et c'est par suffixation GL que l'on en dérive l'antécédant inchoatif, le PP ikusiko (basque standard), mais les dialectes alternent les deux génitifs, le possessif GP (ikusiren) et le locatif GL (ikusiko)22. La B2 admet, comme le nom verbal, les postpositions de la déclinaison nominale, soit directement (ADL ikusira, INSTR ikusiz, ABL ikusitik, GL/GL ikusiko / ikusiren), soit après -a déterminatif de nominalisation ikusia suivi de postpositions, (IN ikusian, E ikusiak, D ikusiari); ceci nécessitera que l'on précise le rôle de -tze sur la B1 (nom verbal ikuste) puisque le PA, lui aussi, est « déclinable». En l'absence de $a$, la fonction de la forme verbale est déterminée par la nature du rapport exprimé par la postposition : les génitifs forment des participes (ikusiko / -ren), l'adlatif et l'instrumental forment des adverbiaux (lehen ikusira «à première vue », ikusiz. « en voyant »). Enfin, les dérivations adjectivales partitive (ikusirik) et résultative (ikusita) «vu» sont l'une et l'autre successivement adnominalisables par une marque de GL -ko (ikusiriko / ikusitako sagua «la souris vue »), puis nominalisable par le déterminatif - a (ikusirikoa, ikusitakoa «celle qu'on a vue »), et enfin prolongeables par des postpositions concaténées (E ikusirikoak / ikusitakoak); au datif pluriel : ikusitakoei buruz 'vu-RES-GL-D-PL tête-INSTR' «à

${ }^{22}$ La Règle 85 de l'académie (Euskaltzaindiaren Araua 85) stipule les conditions de la distribution des deux génitifs pour la formation du participe futur: certains dialectes optent pour l'une ou l'autre forme de manière exclusive, d'autres les font alterner selon des contraintes morpho-phonologiques. On constate cependant la propension de certains locuteurs à user de l'alternance non pas en fonction de critères phonologiques mais selon une distribution fondée sur les degrés de présupposition (Bottineau 2006). Euskaltzaindiaren Araua 85 :

http://www.euskaltzaindia.net/arauak/dok/Araua_0085.pdf 
propos de celles qu'on a vues ». Ces enchaînements sont obtenus par implicitation de substantifs intermédiaires (ikusitako (sagu)ei buruz «à propos des (souris) vues ») et ne constituent pas des surdéclinaisons à proprement parler.

Suite à ce panorama, on détaille à présent les nominalisations et formes nominales repérables aux différents niveaux du tableau: (i) sous B0: les nominalisations des propositions à verbe ou auxiliaire personnel final (section 3.) ; (ii) sous B1 : le NV en -tze et le participe inaccompli en -tzen (section 4.); (iii) sous B2: le PA, ses trois formes adjectivales et leurs dérivations adnominales ou nominales (section 5.).

\section{LA NOMINALISATION DE PROPOSITIONS A VERBE OU AUXILIAIRE MULTIPERSONNEL}

Lorsqu'une proposition à verbe personnel synthétique est nominalisée, adjectivée ou adverbialisée, c'est le verbe final qui agglutine à la racine (B0) les pronoms de l'accord multiple, les déterminations de temps, aspect et allocutivité, et, à leur suite, les marques de nominalisation, adjectivation, et le cas échéant les postpositions «casuelles» pertinente selon la coloration sémantique de la subordination :

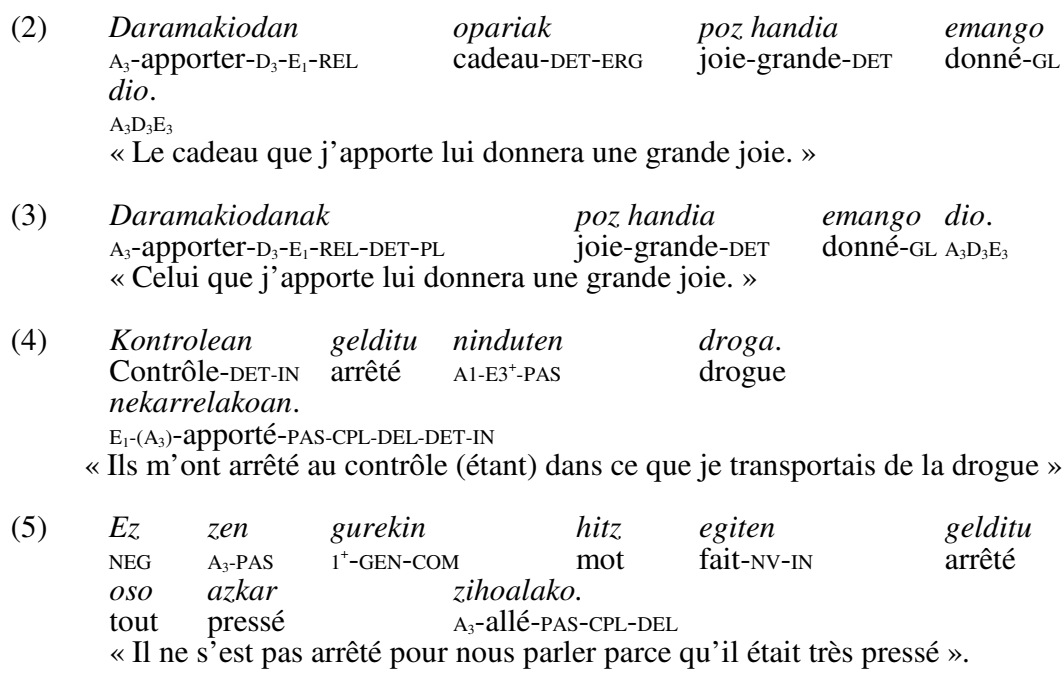

Le même phénomène concerne la $\mathrm{CP}$; c'est l'auxiliaire personnel final qui reçoit en suffixes les marqueurs d'enchâssement propositionnel :

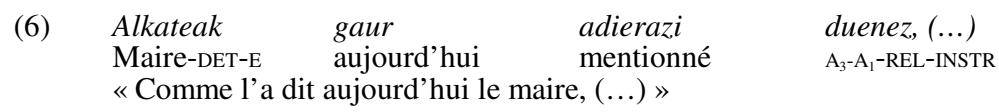


Les compositions courantes formatrices des subordonnées à verbe fini sont nombreuses. Elles s'adossent soient au «conjonctif»-la (marque de discours rapporté formant les complétives des verbes déclaratifs et cognitifs), soit au «relatif»-en (marque de dépendance syntaxique formant les subordonnées interrogatives). Dans tous ces cas, on ne peut parler de forme nominales, adjectivales ou adverbiales du verbe conjugué, mais de propositions enchâssées par nominalisation, adjectivation ou adverbialisation. Le fait que l'affixation concerne toujours le verbe ne doit pas masquer la portée syntagmatique du relateur d'enchâssement, même quand la proposition coïncide avec le verbe seul ; cette affixation porte sur le verbe parce que dans la chaîne syntaxique de la proposition subordonnée celui-ci est toujours final comme il l'est en général dans la proposition indépendante non négative. La proposition conjuguée instancie le groupe lexical du syntagme nominal exactement comme pourrait le faire un simple nom, complété ou non d'un adjectif, d'un adverbe etc. :

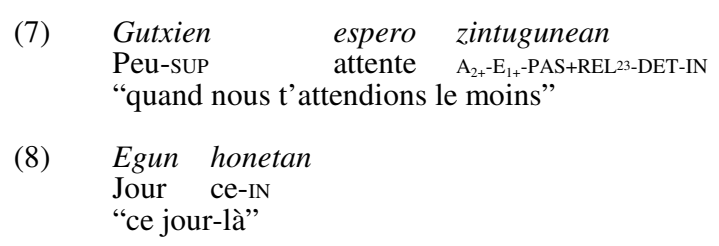

Puisque le paradigme des postpositions (groupe fonctionnel) concerne le groupe notionnel dans son ensemble et n'est réservé à aucune catégorie lexicale en particulier, il n'y a aucune raison théorique que le verbe, conjugué ou non, ne puisse le recevoir s'il se trouve être le dernier constituant lexical du groupe notionnel. Les postpositions sont les marques morphologiques de la valeur syntaxique d'un groupe dans une proposition donnée, elles sont indépendantes de toute catégorisation lexicale concernant le dernier élément affixé. Le segment notionnel, analytique, disjoint des unités lexicales, et le segment fonctionnel, synthétique, conjoint des marqueurs de relation: la structure interne du SN reproduit en partie celle de la proposition.

Cependant il convient de nuancer l'indépendance du contenu propositionnel relativement à la postposition enchâssante: toutes les formes verbales personnelles n'admettent pas avec la même liberté des postpositions subordonnantes. En régle générale, les formes exprimant un ancrage interlocutif immédiat les rejettent. En basque normé ceci concerne (i) l'impératif : emaidazu «donnez-le moi » (*emaidazun, *emaidazula etc.) ; (ii) les formes allocutives :

$\begin{array}{lllll}\text { Etorriko } & \text { ez } & \text { dela } & / * \text { duala } & \text { esan } \\ \text { Arrivé-GL } & \text { NEG } & \text { A3-CONJ } & / * *_{\mathrm{A}_{3}-\mathrm{E}_{2}{ }^{\mathrm{ALL}}-\mathrm{CONJ}} & \text { dit } \\ \text { diet } / & \text { zieat }^{24} & & & \end{array}$

${ }^{23}$ Le - $\underline{\underline{n}}$ - de zintugunean amalgame (i) le $-n$ de passé de la forme à subordonner, zintugun, et (ii) le $-n$ - relatif qui la subordonne en introduisant la nominalisation $(-a-)$ qui supporte l'inessif ( $-\underline{n}$ final).

${ }^{24} z$ ieat $=z-i-e-k a-t$ : l'allocutif masculin $k a$ est réduit à $a$ en position intervocalique. 


$$
\begin{aligned}
& \mathrm{A}_{3} \mathrm{D}_{3+} \mathrm{E}_{1} / \\
& \text { «Je leur ai dit qu'il ne viendrait pas. » }
\end{aligned}
$$

L'auxiliaire principal diet «le-leur-je » admet une version allocutive zieat «leleur-te-je » mais il n'existe pas de «concordance allocutive » en subordonnée complétive de type dela «il-que »> *duala «il-te-que», alors que la même forme allocutive est recevable en indépendante :

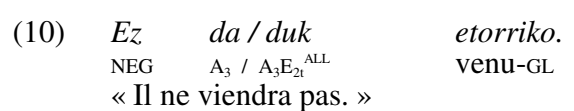

Mais il faut reconnaître qu'en basque actuel (tel qu'il apparaît dans les forums, y compris ceux rédigé dans le batua le plus correct), les locuteurs transgressent très souvent cette prohibition et réalisent la concordance allocutive en subordonnée. De manière révélatrice, les formes du mode potentiel, modalité plus élocutive qu'immédiatement allocutive, admettent la relativisation :

$$
\begin{aligned}
& \text { (11) Ez da edonork egin dezaken zerbait. } \\
& \text { [NEG } \mathrm{A}_{3} \text { ou-qui fait } \mathrm{A}_{3} \text {-SUBJ-HYP-E } 3 \text { ]-REL quelquechose } \\
& \text { «Ce n'est pas quelquechose que n'importe qui peut faire ». }
\end{aligned}
$$

La modalité, subjective, admet un recul subordinatif assumé par le locuteur; l'allocutivité, intersubjective, ne souffre aucun décalage par rapport au présent immédiat de l'interlocution et ne se matérialise que par la prédication principale. Enfin, l'auxiliaire au subjonctif souligne sa dépendance du verbe principal par $-n$, à mettre en parallèle avec le GP aitaren kotxea «papa-de voiture-la » :
(12) Ekipamendu egokia eros dezazun nahi dugu. [équipement adéquat-DET acheté A3-SUBJ-E2]REL volonté $\mathrm{A}_{3}-\mathrm{E}_{1_{+}}$ « Nous voulons que vous achetiez l'équipement adéquat. »

-n marque à la fois le GP nominal, l'inessif (Bilbon “à Bilbao"), les subordonnées relatives (au postcédant), les subordonnées interrogatives (relatives au verbe principal), les subordonnées subjonctives (relatives à la modalité principale), et même les indépendantes au passé (relatives à l'instant de parole et de présent absolu): [ikusi nue]n "je le vis". Cet isomorphisme de catégories hétérogènes peut être fortuit, mais il pourrait également être sous-tendu par une forme schématique commune et transcatégorielle (Robert 2003) déclinant un rapport commun de dépendance analogiquement souligné parmi ses diverses réalisations; et il est frappant de constater une cohérence similaire pour - $k$-, qui partout $s$ 'associe à $-n$ - dans la formation de systèmes binaires ${ }^{25}$ : les génitifs, les

25 Certains de ces systèmes sont des oppositions exclusives, comme $k a$ et $n a$ allocutifs. D'autres forment des contrastes inclusifs: - $n$ (passé) et -ke (hypothèse) sont complémentaires (ikus zezakeen «il pouvait (le)voir»). Certains se combinent différentiellement à d'autres opérateurs (GP + DET - a etxearen vs GL etxeko « de la maison », ce qui complexifie la notion d'opposition, d'autant que les dialectes ont en la 
degrés de l'adjectif (comparatif -ago, superlatif -en), les repérages du verbe personnel (passé - $n$, hypothèse -ke), le genre des marques de l'allocutaire tutoyé (féminin $n a$, masculin $k a$ ); l'exploration de cette voie relève d'un travail ultérieur.

\section{BASE UN}

\subsection{Base $1+$-tze-(ou allomorphes) : le «nom verbal»}

Le NV se forme en ajoutant un suffixe -tze à la B1: ikus > ikuste «le fait / l'action de voir», emate «action de donner», uzte «action de laisser» etc. Creissels (2006) montre qu'en réalité il n'existe pas pour cette forme de dénomination satisfaisante en grammaire traditionnelle et rappelle que certains grammairiens préconisent l'emploi du terme mazdar emprunté à la grammaire arabe (gérondif étant un candidat admissible mais déclassé par l'usage détourné qu'en a fait la grammaire descriptive). En effet, du côté nominal le NV admet l'ensemble des postpositions de groupe nominal : irakurtzeak «le fait de lire » à l'ergatif ; mais du côté verbal, ce même nom verbal admet sur sa gauche ses actants nominaux classiques (absolutif, ergatif, datif) sans modification morphosyntaxique par rapport au verbe personnel de l'indépendante correspondante (sauf dans les dialectes de France, notamment en souletin ${ }^{26}$ ); Creissels donne pour exemples :

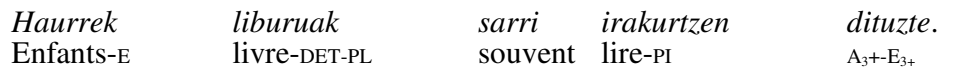

«Les enfants lisent souvent des livres »

$\begin{array}{llll}\text { Haurrek } & \text { liburuak } & \text { sarri } & \text { irakurtzeak } \\ \text { enfants-E } & \text { livre-DET-PL } & \text { souvent } & \text { lire-NV-E } \\ \text { poztu } & \text { egiten gaitu. } & & \\ \text { contenter } & \text { faire-PI } \mathrm{A}_{1+}-\mathrm{E}_{3} & & \\ \text { «Le fait que les enfants lisent souvent des livres nous réjouit. » }\end{array}$

matière des fonctionnements contrastés (dialectes du nord sauf souletin: distribution complémentaire GP / GL; souletin : GP exclusivement ; dialectes du sud - biscayen, guipuscoan : GL exclusivement). Envisager un système $n / k$ sous-jacent n'implique pas le nivellement des couples d'opérateurs impliqués, les situations sont diverses.

26 En souletin «l'objet » du NV est au GP et non à l'absolutif : Haren ikustera noazü 'luide / voir-à / je-vais-vous' = 'je vous vais à son voir' (allocutif) > «je vais le voir »; Horren egitez irabaziko düzü ' ç̧a-de / faire-par / gagner-de / le-vous' = 'vous (le) gagnerez par le faire de cela' > «en faisant cela vous gagnerez»: la portée de la nominalisation se limite au verbe lexical comme en français pour les noms d'action déverbaux transitifs en -tion, -ment, -age, -ure etc. (la construction de l'autoroute, le traitement de la maladie, le partage du butin, la capture du fugitif), le NV anglais (the writing of the book «l'écriture du livre ») et breton (Ret eo e lenn 'Dû est son lire' > « Il faut le lire »), etc. 
A titre de comparaison, l'anglais présente bien des cas de prédicats transitifs nominalisés sans préposition devant l'objet (this crossing the Alps, exemple de Jespersen) mais le sujet sera soit possessif (John's / his crossing the Alps), soit régi par un verbe (I saw John / him / *he crossing the Alps), jamais autonome comme l'est ici l'ergatif basque. Tout se passe comme si -tze- neutralisait la synthèse attendue des arguments lexicaux dans l'auxiliaire (conjugaison périphrastique) et confinait l'ensemble propositionnel verbe + arguments dans un segment notionnel d'ordre supérieur, lequel sera suivi d'un segment fonctionnel de postpositions assignant au tout un rôle syntaxique dans la proposition enchâssante - en l'occurrence, l'ergatif déterminé [[... irakur]-tze]-ak. Cette analyse est corroborée par le fait que -tze neutralise toute contrainte de coréférence individuelle entre les arguments de la subordonnée et ceux de la principale. Dans l'exemple de Creissels, irakurtze «lire» a pour arguments haurrek «les enfants» et liburuak «les livres», alors que poztu «plaire» (ici focalisé par egiten «faire », PI) a pour arguments la proposition clôturée par irakurtze et un $g u$ «nous » implicite repris dans l'agglutination auxiliée gaitu «nous-ça »: gaitu n'incorpore aucun des arguments individuels rattachés à irakurtze. Dans ce cas, la structure ne peut être rendue par un infinitif roman : *les enfants lire des livres nous plaît vs Lire des livres nous plaît, correct à cause de la coréférence liant individuellement l'agent implicite de l'infinitif au complément explicite de plaît. Evidemment une telle coréférence est très possible et courante en basque, auquel cas la traduction infinitive est recevable :

$\begin{array}{llll}\text { Filme hori ikusteak } & \text { bizkortzen } & \text { nau. } \\ \text { film ce } \quad \text { voir-NV-E } & \text { réconforter-PI } & \mathrm{E}_{1}-\mathrm{A}_{3} \\ \text { «Voir ce film me réconforte. » } & & \end{array}$

Il arrive qu'un énoncé commence par deux ergatifs consécutifs, l'un lié au verbe final de la principale, l'autre au «nom verbal » de la subordonnée :

$\begin{array}{lllll}\text { Nik } & \text { haurrek } & \text { liburua irakurtzea } & \text { nahi } & \text { dut. } \\ \text { 1-E } & \text { enfant-DET-PL-E } & \text { livre-DET lire-NV } & \text { vouloir } & \mathrm{A}_{3}-\mathrm{E}_{1}\end{array}$

«Moi je veux que les enfants lisent le livre. »

Toutes les déclinaisons sont compatibles avec le NV (Bottineau 2006). Le GL en $-k o$, en particulier, permet (i) de rendre la subordonnée adnominale : ezetz esateko moduak «les manières de dire non »; (ii) de compléter un adjectif: Jakina, prest nago laguntzeko «Bien sûr, je suis prêt à aider »; (iii) de compléter un verbe type «demander», «ordonner»: Zu ere bai etorri zara, eta ez etortzeko esan dizut. «Toi aussi tu es venu, et je t'ai dit de ne pas venir. »Ces constructions, syntaxiquement distinctes, partagent une valeur sémantique : -ko indique que l'action subordonnée est sélectionnée par le sujet au sein d'un ensemble de possibilités - «comment s'y prendre pour dire non (plutôt que oui) », « être prêt à aider (plutôt que tirer au flanc) », « dire à quelqu'un de faire quelque chose (plutôt qu'autre chose) ». Pour les locuteurs qui dans leur dialecte 
pratique une opposition pertinente, cette valeur fonde également le contraste entre les deux génitifs du PP (Bottineau 2006).

\subsection{Le «nom verbal » ̀̀ « l'inessif archä̈que » : le participe inaccompli ikusten}

Le NV sert de support à la formation du PI : ikuste + - $n=i k u s t e n$ «voyant », que l'on analyse traditionnellement comme une forme archaïque de l'inessif indéterminé (distincte de l'inessif déterminé, hori ikustean "voyant cela »). Les trois emplois principaux sont la CP (4.2.1.), la périphrase verbale d'inaccompli en ari «agir» (4.2.2.) et la complémentation des verbes semi-auxiliaires, aspectuels, cognitifs, triadiques (type laisser), d'effort (type essayer), de mouvement et de complémentation adjectivale complexe (4.2.3.) (Hualde \& Ortiz de Urbina 2003, 674-689).

4.2.1. Le PI se combine librement aux auxiliaires des diverses valences pour l'expression de l'aspect itératif : egunkaria irakurtzen dut «je lis le journal» (générique ou spécifique selon les contextes), «je me place dans la lecture du journal » (non spécifiquement située, donc pouvant être générique). Comme en témoigne l'accord multiple de l'auxiliaire final $d u t$ (E et A), le PI ne prend pas les arguments nominaux pour son propre compte comme en (15) et (16): si la portée de -tze du NV est propositionnelle et inclut les arguments, celle de -tzen du PI est lexicale, concerne le verbe seul et s'apparente à une flexion, tout comme les GL - ko et GL -en des PP.

4.2.2. Cette suggestion est confortée par l'existence d'une périphrase verbale intransitive en PI + ari, comparable à être en train de. Ari s'utilise selon les dialectes comme adjectif régissant un gérondif, comme particule aspectuelle ne modifiant pas la valence verbale, et comme prédicatif intransitif. Ce dernier emploi souligne l'aspect inaccompli singulatif avec des implications contextuelles, situationnelles ou interlocutives ressortissant à la modalité (proche de l'anglais be $+\mathrm{V}$-ing) :

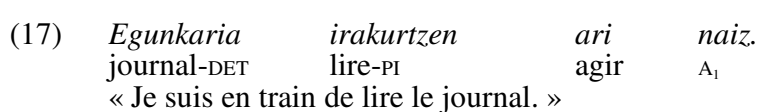

L'auxiliaire uniactanciel naiz s'accorde avec le verbe uniactanciel ari «agir », la glose étant «j'agis dans la lecture du journal (donc ne me dérange pas)»; en soulignant l'action de l'agent considéré isolément, ari l'oppose à celle d'agents co-occurrents en situation, avec de multiples implications pragmatiques. L'absolutif egunkaria n'est lié qu'à irakurtzen et ne laisse aucun accord dans naiz. L'erreur d'analyse serait probablement de dire que irakurtzen contrôle l'ensemble des arguments qui précèdent; c'est cette fois ari qui a l'effet de verrouillage ou «d'imperméabilisation » bloquant l'incorporation des arguments initiaux à l'auxiliaire final : la structure est bi-propositionnelle. Dans les dialectes du sud le verbe egon «être » (esp. estar) peut être utilisé à la place de ari + AUX : 
4.2.3. Pour les classes de verbes à complément inaccompli, abondamment illustrées par Hualde et Ortiz de Urbina, la même contrainte de coréférence est observée :

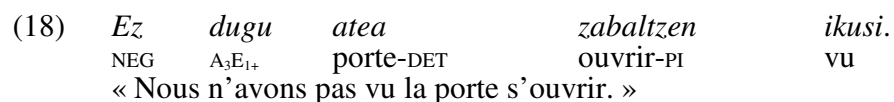

L'absolutif explicite du verbe principale peut coréfèrer à un ergatif implicite du même participant pour le participe inaccompli :

(19)

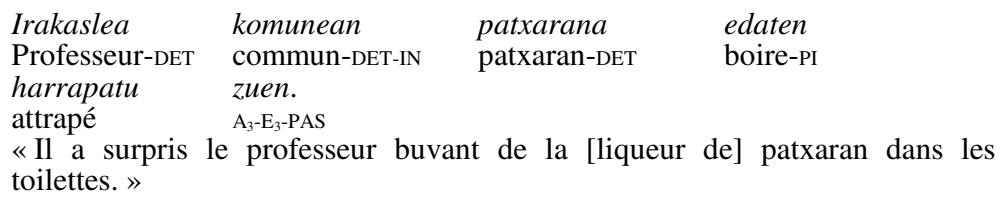

L'explicitation de l'agent buveur passerait par le pronom réfléchi bera «luimême » à l'ergatif berak, mais elle n'est pas possible avec une structure mécaniquement coréférencielle comme harrapatu «attraper» + patient + inaccompli. Avec un verbe triadique :
(20) Legeak ez $\quad$ digu Loi-DET-E NEG $\quad \mathrm{A}_{3}-\mathrm{D}_{1+}-\mathrm{E}_{3}$ autobus-DET-IN «La loi ne nous permet pas de fumer dans l'autobus. »
erretzen uzten. fumer-PI laisser-PI

Hualde et Urbina signalent d'une part une exclusion mutuelle du PI (ikusten) et du NV à l'inessif déterminé (ikustean), la possibilité de l'un proscrivant celle de l'autre, et d'autre part quelques cas où malgré tout le premier alterne avec la forme absolutive du second, sans qu'on puisse décider si l'alternance est stylistique et/ou dialectale. Or notre analyse semble de nature à trancher le contraste (y compris sur leurs propres exemples) : d'une part, le NV permet un enchâssement propositionnel libre de contraintes sémantiques, alors que le PI est régi par une liste de classes verbales parfaitement déterminée au niveau du verbe recteur dont le fonctionnement est proche de celui d'un modal ; le premier est bipropositionnel, le second mono-propositionnel. D'autre part, l'alternance entre les deux est rendue possible par l'opposition référence / non-coréférence : pour citer leurs exemples, dans

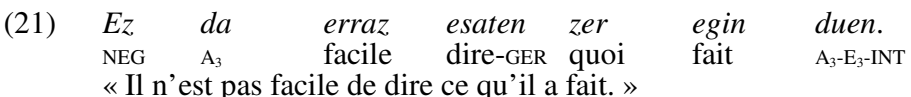

esaten «dire » a pour agent implicite un locuteur qui coïncide avec celui de la principale, implicite lui aussi, ce qui se glose «il ne m'est pas facile de dire ce qu'il a fait ». Au contraire, dans
(22)
Hori erraz da
esatea, zailago
egitea. 
Ceci facile $\mathrm{A}_{3}$ dire-NV difficile-plus faire-NV

«Il est facile de dire ça, plus difficile (le) faire »

(«C'est plus facile à dire qu'à faire»).

l'agent implicite de esatea « dire », générique ou renvoyant à un ou des individus déterminés par la situation ou le contexte, ne coïncide pas avec le locuteur qui, justement, exprime un point de vue personnel non solidaire de celui des autres ; ou du moins ne rapporte-t-il pas la source du dire à lui-même exclusivement. Le PI est réflexif (par opposition à obviatif) et s'appuie sur un sujet mémorisé, alors que le nom verbal, indéterminé en la matière, est ouvert à l'inclusion d'autres acteurs. Cette structure permet des enchâssements très concis :

(23)

\begin{tabular}{|c|c|c|c|c|c|}
\hline $\begin{array}{l}\text { Gaur egun } \\
\text { Aujourd'hui }\end{array}$ & $\begin{array}{l}z e r \\
\text { quoi }\end{array}$ & $\begin{array}{l}e z \\
\text { NEG }\end{array}$ & $\begin{array}{l}\text { ikusteak ematen } \\
\text { voir-NV-E donner-PI }\end{array}$ & $\begin{array}{l}\text { dizu } \\
\mathrm{I}_{3} \mathrm{D}_{2} \mathrm{E}_{3}\end{array}$ & $\begin{array}{l}\text { pena? } \\
\text { peine }\end{array}$ \\
\hline $\begin{array}{ll}\text { Gure } & \text { arteko } \\
1+-\mathrm{GP} & \text { entre-GL }\end{array}$ & $\begin{array}{l}\text { elkar } \\
\text { RCP }\end{array}$ & com & $\begin{array}{l}k \\
\text { dre-NV-PART }\end{array}$ & $\begin{array}{l}e z \\
\text { NEG }\end{array}$ & $\begin{array}{l}\text { ikusteak. } \\
\text { voir-NV-E }\end{array}$ \\
\hline
\end{tabular}

5. BASE 2

\subsection{Le participe accompli avec postpositions}

La B2 ikusi «voir » signifie l'actualisation de l'ensemble du procès verbal, aussi est-elle accompagnée des formes de l'auxiliaire « indicatives ${ }^{27}$ impliquant la réalité du procès : le présent ikusi dut «je l'ai vu», le passé ikusi nuen «je le vis », le conditionnel ikusiko nuke « je le verrais », le conditionnel passé ikusiko nukeen «je l'aurais vu», par opposition aux formes virtualisatrices accompagnées de la base un comme le potentiel ikus dezaket «je peux le voir». Ces formes impersonnelles sont considérées comme verbales: le verbe est l'ensemble périphrastique monopropositionnel formé par le couple B2 + auxiliaire ; s'il fallait analyser la structure comme une forme verbale nominale dépendant de l'auxiliaire, alors cette base deux entrerait dans la valence même de l'auxiliaire (comme le fait le NV) et serait comptabilisée comme argument absolutif, rendant impossible l'adjonction d'un autre nominal absolutif pour exprimer le patient ou le site; or on a bien herri hura ikusi dut «j'ai vu cette ville », l'auxiliaire dut «la-je » renvoyant à l'absolutif herria «ville-la » et à l'ergatif nik «moi-E », ikusi étant actanciellement neutre. En outre, la B2 sert de support à la dérivation génitive des participes futurs : ikusi $+k o=i k u s i k o, i k u s i$ + ren = ikusiren. Là aussi se pose la question de la nominalité d'une forme verbale génitivable. Il n'en est rien: comme pour ikusi on trouve ikusiko /

${ }^{27}$ L'usage des catégories romanes pour le basque est généralement à proscrire, mais pour l'objet qui nous intéresse elle n'est pas préjudiciable, aussi se permettra-t-on ce raccourci. 
ikusiren dut « je le verrai » où l'auxiliaire incorpore des pronoms renvoyant à des arguments nominaux distinct du verbe. Il s'ensuit que ikusiko et ikusiren doivent être analysés comme des variables flexionnelles du paradigme verbal de ikusi et que les marques génitives marquent un rapport de dépendance sémantique entre le procès et l'auxiliaire signifiant sa réalisation, d'où un effet de sens de projection ou de planification de type haber de espagnol (mais de coloration modale beaucoup plus faible) $)^{28}$.

Pourtant la base deux ikusi se prête elle aussi à la nominalisation propositionnelle : on distingue ikuste «action de voir » et ikusi «chose vue »; ikustea «l'action / le fait de voir » et ikusia « (la chose) vue », «(ce qui) est vu ». La distinction entre nom et adjectif n'est pas pertinente : dans la structure de forme sujet + «être » + attribut, le sujet et l'attribut, nominal ou adjectival, reçoivent le même morphème déterminatif $-a$, andrea polita da « la femme est jolie » / zakurra animala da «le chien est un animal». De ce fait, ikusia se rencontrera dans des environnements de type adjectival ou nominal :

Adjectival :
(24)

$\begin{array}{llr}\text { Igerilekua } & \text { barrutik } & \text { ikusia } \\ \text { Nage-lieu-DET } & \text { intérieur-ABL } & \text { vu-DET } \\ \text { «[Bergara : ] la piscine, vue de l'intérieur » }\end{array}$

Nominal :

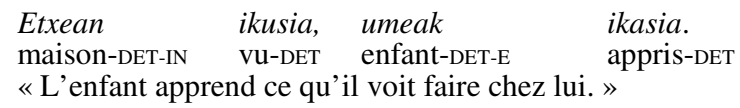

Comme pour le NV, le PA clôturant une proposition (i) reçoit la postposition (E, $\mathrm{D}, \mathrm{IN}$ etc.) qui affecte à cette proposition un rôle syntaxique dans le proposition où elle s'intègre,

$$
\begin{aligned}
& \text { Eta hark dioenez, bere ingurumenean ikusiak } \\
& \text { et 3-E } \mathrm{A}_{3} \text {-dire-E } \text { E }_{3} \text {-INSTR son alentour-DET-IN vU-DET-PL } \\
& d u \text { inspiratu. } \\
& \mathrm{A}_{3}-\mathrm{E}_{3} \quad \text { inspiré } \\
& \text { «Et d'après ce qu'il dit, c'est ce qu'il voit dans son environnement qui } \\
& \text { l'inspire. » [Le verbe est focalisé par l'inversion avec l'auxiliaire] }
\end{aligned}
$$

et (ii) B2 ikusi, à l'intérieur de sa propre proposition, est précédée de ses propres arguments (A, D, E) :

(27) Mikaela bai poztuko dala zu $\quad$ ikusiarekin.

${ }^{28}$ Cf. le début de la $3^{\text {e }}$ églogue de Garcilaso de la Vega: El dulce lamentar de dos pastores / Salicio y juntamente Nemoroso / he de cantar... «La douce plainte de deux bergers / Salicio et avec lui Nemoroso / j'ai à chanter... » 
«Bien sûr que Mikaela sera contente de vous voir. »²9

$\begin{array}{cccc}\text { (...) berak ikusiari ta } & \text { ikasiari } & \text { dagokio. } \\ \text { 3-E } & \text { vu-DET-D et } & \text { appris-DET-D } & \text { A }_{3} \text {-être- } \mathrm{D}_{3}\end{array}$

Les postpositions ajoutables à ikusi sont énumérées dans Bottineau 2006. Il apparaît que la compatibilité de la B2 ikusi avec les différentes postpositions ne diffère en rien de celles que l'on avait illustrées pour le NV ikuste. On retrouve par là même notre propos initial, à savoir que la nominalisation concerne l'ensemble du syntagme, la proposition, mais s'accroche au dernier élément, le verbe, du simple fait qu'il se trouve typologiquement fixé en cette position et non en vertu d'une supposée nominalité de la forme verbale.

Entre ikuste et ikusi, la différence morphologique est la suivante : le NV porte un suffixe qui le réserve à la fonction de «queue verbale impersonnelle » d'une proposition nominalisée par enchâssement, contrairement à la B2, ikusi, que rien ne différencie formellement d'un participe tant que l'affixation des postpositions n'a pas eu lieu. Ikuste reste une forme de langue, par opposition à ikusi, qui connaît les mêmes emplois en discours (au sens de l'opposition guillaumienne entre langue et discours en psychomécanique du langage) ; ikuste est une forme réservée, ikusi non. Enfin, le nom verbal basque connaît des homologues catégoriels indo-européens tels que le nom verbal en -ing de l'anglais qui facilitent sa lecture nominale par les linguistes, alors que les équivalents de la base deux sont plutôt adjectivaux ou relatifs par rapport au déterminant $-a$ : ikusia «le vu», «la chose vue», «ce qui a été vu», «le fait d'avoir vu». On pourrait penser que le déterminant $-a$ joue un rôle moteur dans la déverbalisation du PA, mais certains cas spatiaux le proscrivent (GL, PART, ADL, ABL), et l'instrumental admet l'indétermination (ikustez vs ikusteaz); seuls l'inessif, le génitif possessif, l'absolutif, l'ergatif et le datif le requièrent. On ne peut pas défendre que - $a$ soit pour la B2 un substitut discursif de -tze de langue pour la B1 dans la fonction de clôture propositionnelle.

Il semble donc raisonnable de proposer la solution suivante : le basque dispose de formes de langue bien distinctes, d'une part une forme générale, la B2 ou PA, compatible en discours avec la fonction de queue verbale impersonnelle d'une proposition nominalisée enchâssée par les postpositions, même si ce n'est pas la seule fonction possible de la $\mathrm{B} 2$; et d'autre part, le NV, forme de langue dérivée de la $\mathrm{B} 1$, réservée à la fonction de queue verbale impersonnelle. A partir de formes de langue distinctes, une mise en convergence des structures discursives

29 Pour (27) la structure est habituellement à l'instrumental, du moins en contexte présent et quand le sentiment exprimé est celui du locuteur : atsegin handi dut zure ikusteaz 'plaisir grand le-je [votre voir]-par' «J'ai grand plaisir à vous rencontrer ». (27) utilise le comitatif -ekin (accompagnement / causalité) pour un sentiment doublement projeté, dans le temps (poztuko da «sera contente ») et l'intersubjectivité (Mikaela) : dans mon corpus d'exemples l'instrumental s'applique à un procès évalué par le locuteur; en cas de délégation de point de vue l'écart est souligné par le comitatif. Ceci semble conforme aux vues de Ducrot sur la polyphonie. 
compatibles (ikusi) ou réservées (ikuste) neutralise la distinction morphosyntaxique sous-jacente. Ceci fait apparaître dans l'usage des effets de paradigmes discursifs alternant des formes de niveaux constructionnels distincts en langue ; on a constaté le même type de rapprochement entre les trois participes (accompli, inaccompli, prospectif) vis-à-vis de l'auxiliaire personnel final alors même que ces participes résultent morphologiquement de niveaux constructionnels hétérogènes (B2, B1dérivée, $\mathrm{B} 2$ dérivée).

\subsection{Formes résultatives}

La B2 ikusi connaît encore quelques emplois difficilement classables en termes de catégorisation. Le basque distingue en tout quatre formes équivalant au participe passé roman ou germanique. A lui seul, le PA marque la réalisation intégrale de l'événement marqué par le verbe, et ce jusqu'à l'obtention du résultat: Film bat ikusi dut «j'ai vu un film », Bi film ikusi ditut «J'ai vu deux films »; la forme de l'auxiliaire varie par l'accord multiple à «l'objet » absolutif (singulier vs pluriel) mais celle du participe, d'ordre verbal et non nominal, ne varie pas. Mais ce participe peut être augmenté de trois morphèmes au choix : le déterminant - $a$ : ikusia (5.2.1), le partitif -rik (adverbial) : ikusirik (5.2.2), et le résultatif -ta: ikusita (5.2.3), les deux derniers pouvant être rendus adnominaux par -ko (ikusiri(ka)ko, ikusitako. L'analyse de leur distribution pose le même problème que les formes GL et GP : les dialectes du sud utilisent -tako, ceux du nord alternent des formes en -riko et -rikako en alternance avec le PA; la valeur des alternances est à spécifier au cas par cas. Dans notre propre corpus (traductions en batua de Harry Potter $^{30}$ et de bandes dessinées en grand nombre), il apparaît une distribution cohérente que nous présentons ici; on peut la considérer opératoire pour les idiolectes concernés faute d'être pleinement généralisable.

5.2.1. -Le PA déterminé ikusia. - La marque de détermination - $a$ signale que dans un syntagme le segment notionnel, par exemple un nom etxe «maison », est relié par le segment fonctionnel, $-a$, à un référent repérable (dans l'extralinguistique, le discours, la mémoire, etc.) : etxea « une / la maison », etxea $d a$ «c'est une / la maison ». Ce morphème est complétable du pluriel - $k$ : etxeak «des / les maisons », etxeak dira «ce sont des / les maisons ». Ces mêmes morphèmes s'appliquent à l'adjectif attribut stabilisant une propriété repérée du sujet : Etxea zuria da « la maison est blanche », Etxeak zuriak dira « Les maisons sont blanches » (litt. 'les choses maison et les choses blanches forment un'). Appliqué au PA, le déterminant - $a$ convertit l'accompli d'événement en propriété stabilisée du sujet au même titre que celle exprimée par un attribut: [Film hau ikusi]a naiz «J'ai vu ce film», «Je suis une personne ayant vu ce film ». Cette structure est très proche de l'antipassif, lequel utilise le résultatif $i k u s i t a$ en lieu et

30 Dans Harry Potter, une exception : l'anglais dialectal de Hagrid est systématiquement traduit en guipuscoan. 
place du déterminé $i k u s i a^{31}$; la différence sémantique a trait aux implications modales du résultatif (5.2.3. infra). Par contre elle n'a rien à voir avec le «passif» (5.1. supra), dont l'absolutif initial contrôle directement l'auxiliaire sans être incorporé à une subordonnée clôturée par ikusia: Film hau ikusia da (nik) «ce film a été vu (par moi) ». En contexte :

$$
\begin{array}{llll}
\text { (29) Jakin ezak ni } & \text { gerran } & \text { ibilia } & \text { naizela! } \\
\text { savoir } \mathrm{A}_{3} \text {-IMPER-E } \mathrm{E}_{2} 1 & \text { guerre-DET-IN } & \text { marché-DET } & \text { A }_{3} \text {-CONJ } \\
\text { «Sachez que je suis allé à la guerre! » } & \\
\text { Gergovian! Zer } \quad \text { uste duk } & \text { hik? } \\
\text { Gergovie-IN quoi croyance } \mathrm{A}_{3}-\mathrm{E}_{2 \mathrm{t}} & \text { 2t-E } \\
\text { «A Gergovie ! Qu'est-ce que vous croyez ? } &
\end{array}
$$

Le protestataire s'auto-définit comme un ancien combattant bénéficiant de la gloire présente afférant à son statut. Ce verbe intransitif ibili «marcher » ne laisse qu'une construction possible, mais avec un verbe transitif comme ikusi le participe ikusia pourra être accompagné soit de l'auxiliaire intransitif, ikusia naiz, littéralement «je suis vu », ne focalisant que l'état résultant postérieur au procès ; soit de l'auxiliaire transitif, ikusia dut «j'ai vu », exprimant d'une part l'opérativité du procès (ikusi dut) et d'autre part la stabilisation de l'accompli (a) comme propriété du sujet considéré comme témoin ou de l'objet posé comme fait établi. On le traduit alors habituellement par un aspect parfait, typiquement le plus-que-parfait en contexte passé (ikusia nuen «j'avais vu»), alors que le contraste n'apparaît pas au présent. Si on explicite tous les arguments nominaux, dans le premier cas on a deux absolutifs, le premier renvoyant au verbe personnel final, le second au participe accompli déterminé (structure bi-propositionnelle) : $N i$ \{film hau ikusi\}a naiz «je suis ayant vu ce film»; dans le second cas on a deux arguments, l'un ergatif, l'autre absolutif, renvoyant ensemble à l'auxiliaire final au sein d'une proposition unique : Nik film hau ikusia dut «j'ai vu ce film ». L'exemple suivant (dont on omettra la translittération) est particulièrement éclairant en la matière. Au cours d'une partie de Quidditch (sport de sorciers), Harry Potter sur son balai voit la balle magique échapper de la main d'un des joueurs (on restitue la version anglaise d'origine) :

(30) a. Harryk ikusi zuen. Erabateko zirratzaz, goitik behera abiatu zen ziztu bizian urrezko arrastoaren atzetik. Slytheringo bilatzaile ere ikusia zuen. Elkarren pare-parean joan ziren snitcharren bila... bazirudien jazarle guztiei ahaztu zitzaiela beren eginbeharra, airean zintzilika baitzeuden begira. $b$. Harry saw it. In a great rush of excitement he dived downwards after the streak of gold. Slytherin Seeker Terence Higgs had seen it, too. Neck and neck they hurtled towards the Snitch - all the Chasers seemed to have

31 Coyos (2002 et 1999, 368) distingue les parcours actif, passif et antipassif en fonction de la covariation de la marque de l'agent, de l'auxiliaire sélectionné, de la flexion participiale, et de l'accord (simple ou multiple). Dans le dialecte souletin qu'il décrit l'agent antipassif peut être marqué par l'ergatif ou l'instrumental, et le suffixe résultatif est -ik (cf. le partitif de même forme). 
forgotten what they were supposed to be doing as they hung in mid-air to watch.

Ikusi zuen est narratif: on a dans cet ordre la vision puis la réaction; ikusia zuen est attributif et fait de Terence Higgs un "ayant vu" déjà élancé à la poursuite de l'objet, ce que confirme la phrase suivante. Ikusia zen serait inadapté ici, faisant de Higgs un dépositaire inactif de l'expérience passée «avoir vu » non impliqué dans la poursuite de l'action. Ce qui surprend dans ce système c'est d'une part l'indifférenciation entre les formes purement verbales ou nominales, puisqu'on trouve ikusi dut (pas d'accord, auxiliaire transitif), ikusia $d u t$ (accord, auxiliaire transitif) et ikusia naiz (accord, auxiliaire intransitif) ; et d'autre part la prolifération des combinaisons structurellement possibles et sémantiquement pertinentes, chaque micro-système indépendant fonctionnant localement pour son propre compte tant que sa production demeure componentiellement compatible avec celle des autres.

5.2.2. - Le PA partitif adjectival ikusirik. - Le partitif -rik adverbialise le PA : cette forme est n'est compatible qu'avec une matrice actancielle (absolutif seul) ; l'adjectif peut être rendu adnominal (équivalent à un épithète) moyennant l'ajout du GL - ko, ce qui donne -riko (élimination de la gémination par amalgame des vélaires, conformément à une contrainte morpho-phonologique universelle en basque). Le partitif quantifie la propriété observée, aussi est-il le plus souvent accompagné du verbe de localisation circonstancielle egon (équivalent à peu près à estar castillan). Cette forme implique une prise de conscience par l'observateur et livre une première évaluation de la qualité découverte. Selon qu'il s'agit de monologue réflexif ou de dialogue transitif, la prise de conscience est adressée soit à soi-même pris pour allocutaire bouclé, soit à autrui, avec une visée didactique prononcée.

$$
\begin{array}{lll}
\text { Baina... } & \text { Baina loturik } & \text { nago ! } \\
\text { mais } & \text { mais attaché-PART } & \mathrm{A}_{1} \text {-être } \\
\text { «Mais... } & \text { Mais je suis attaché ! » } &
\end{array}
$$

(32) a. Eguzkitan jartzen dugunean, eguzki-izpiek beroturik, barneko airea zabaldu egiten da, eta horregatik gogorrago topatzen dugu putxiga. Frigorifikoan, aldiz, hoztu egiten da eta errazonamendu berdinari jarraituz, zimurtu behar dela ikusiko dugu.

$b$. «Quand nous la plaçons [la baudruche] au soleil, l'air qu'elle contient, chauffé par les rayons, se dilate; c'est pourquoi nous la trouvons plus résistante. »

5.2.3. Le PA résultatif ikusita. - Inversement on trouve la forme résultative munie du suffixe - $t a$ (non nominal et invariable en nombre) exprimant la reprise d'un résultat acquis en contexte, d'où le processus d'acquisition ne fait pas mystère. La valeur informationnelle n'est ni la prise de conscience (PART -rik), ni l'établissement assertif de la propriété (DET - $a$ ), mais sa présupposition, sa prise en compte comme fait établi découlant mécaniquement de la situation ou du contexte et ne souffrant aucune discussion: txundituta geratu zen «il resta abasourdi » (on sait très bien pourquoi) vs txunditurik geratu zen (même 
traduction, mais la réaction du personnage constitue une surprise dans le récit). Pour l'opposition entre les adnominaux en -riko et -tako, dans la version basque de Harry Potter à l'école des sorciers (Harry Potter eta sorgin-harria, « HP et la pierre philosophale »), «la fenêtre éclairée » se dit très majoritairement argitutako leihoa, à chaque fois qu'il s'agit d'un élément descriptif allant de soi en contexte (il est normal que la fenêtre soit éclairée), et argituriko leihoa dans la minorité de cas, où manifestement l'auteur attire l'attention du lecteur sur une anomalie, créant interrogation et suspense autour de l'identification de la personne ou du monstre impliqué; ce contraste se vérifie sur des dizaines d'alternances analogues construites sur d'autres participes. Si la forme partitive stigmatise l'inconnu pour le locuteur ou l'allocutaire (avec en ce cas décalage des savoirs préalablement détenus), la résultative au contraire tend à souligner le savoir partagé par les interlocuteurs, invoquant la culture coréférencielle générale ou particulière, avec bien souvent des effets de sens complices (où l'on retrouve des éléments de la théorie des phases d'Adamczewski 1982) :

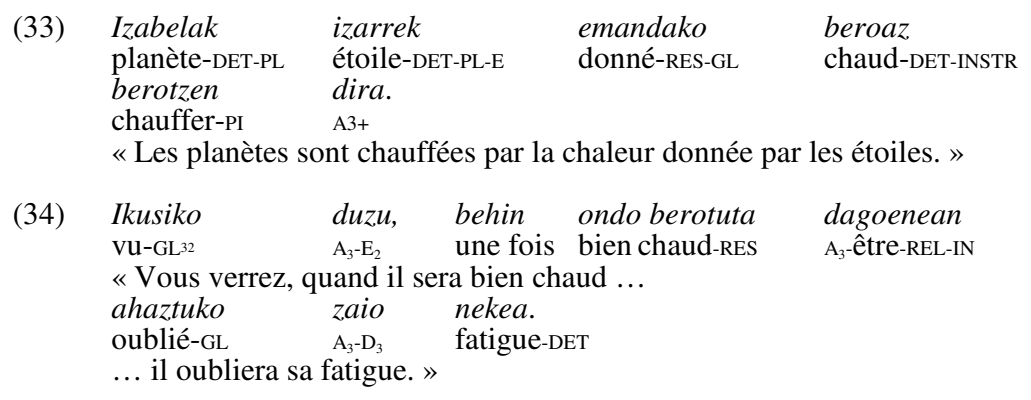

\subsubsection{Interprétation de l'ensemble du microsystème}

Le modèle qui suit constitue un microsystème : il s'applique à des formes dont il est vérifié sur corpus qu'elles alternent effectivement dans les idiolectes respectifs des locuteurs concernés avec les valeurs sémantiques proposées, identifiées en contexte. On observe un dispositif très riche selon lequel l'accompli peut être simplement impliqué par accomplissement intégral (B2 ikusi), découvert par quantification (B2 + partitif ikusirik), asserté / constitué / établi établi par détermination (ikusia) et présupposé ou réévalué par préconstruction (ikusita). La première forme, la B2 nue, est «modalement aoristique » en ce qu'elle pose un accompli indépendemment de tout point de vue subjectif soumis à l'allocutaire par le locuteur-observateur. Les trois autres échelonnent les trois phases psychologiques de l'appréhension subjective de cet accompli : son inchoation (la prise de conscience, la quantification heuristique par le partitif), son exécution (l'établissement, la certification, la validation par le

\footnotetext{
32 Pour $-k o=$ GL : cf. notes 4 et 14 .
} 
déterminatif) et son achèvement (la présupposition, la réévaluation qualitative par le résultatif terminal). Une modélisation guillaumienne du dynamisme cognitif postulé passe par une tension cinétique munie de trois seuils cardinaux d'interception, mais on peut également se référer au trimorphe de Pottier 1992 (ce que fait Coyos 1979 pour d'autres systèmes grammaticaux) et à la sinusoïde de Toussaint 2003 (appliquée par l'auteur à l'opposition ergativité / accusativité).

Ce modèle est complexe parce qu'il fait interagir deux systèmes aspectuels distincts : l'un articule les trois saisies possibles du déroulement du temps d'événement, à savoir le début (ikusiko, PP), le milieu (ikusten, PI) et la fin (ikusi, B2, PA) ; et parmi ces trois seuils, le dernier, et lui seul, la B2 ikusi, fait l'objet soit d'une expression amodale de l'accompli (ikusi), soit d'une appréhension modalisée elle-même décomposée en trois étapes, à savoir l'heuristique (partitif), la pragmatique (déterminatif) et l'herméneutique (le résultatif final). La même tripartition est appliquée à deux domaines distincts, à savoir la mesure du déroulement objectif de l'événement et celle du déroulement subjectif de sa capture par le sujet psychologique. On constate que le triphasage modal n'est compatible qu'avec l'accompli (ikusi) et ne se trouve pas aux deux autres aspects (ikusten, ikusiko, lesquels n'admettent pas les expansions partitive et résultative (*ikusikorik, *ikusikota, *ikustenik, *ikustenda) : le système a beau être très ouvert et productif, il ne permet pas de tout générer de manière non sélective.

Une explication sémantique consisterait à supposer que la quantification subjective ne fonctionne qu'au terme de la quantification objective et que les deux ne peuvent être réalisées concomitamment. Une explication morphologique tient au fait que la forme nue, de base, est précisément ikusi, dont les formes dérivées ikusiko et ikusten sont des amonts interceptifs aspectuels et les formes dérivées ikusirik, ikusia, ikusita des avals modaux : ikusi est la forme lemmatique par défaut au centre du système où elle joue un rôle pivot. C'est parce que la forme perfective est la forme par défaut en basque que l'on peut en dériver des amonts aspectuels objectifs et des avals aspectuels subjectifs ; ces possibilités sont bloquées dès lors qu'une marque aspectuelle prospective ou inaccomplie est posée : il ne s'agit pas de composition cumulative, mais de bifurcation.

\section{CONCLUSION}

La notion de forme nominale du verbe prend un relief très particulier en basque. Dans le domaine nominal, les catégorisations lexicales se jouent par la place de l'unité lexicale relativement aux autres dans le segment notionnel du syntagme nominal, et ce segment reçoit une fonction syntaxique d'actant ou de circonstant par les postpositions constituant le segment fonctionnel, si bien que la morphologie affecte l'ensemble du segment notionnel sans se soucier de la catégorisation lexicale de la dernière unité dans le groupe. Il s'ensuit que pour le domaine du nom, on est obligé d'envisager deux niveaux de catégorisation, l'un de l'unité lexicale par sa place, l'autre du syntagme notionnel par le segment fonctionnel. Cette organisation tient au caractère analytique du domaine nominal, qui discrimine une série de participants en les décomposant en acteurs 
sémantiques (le segment notionnel) couplés à des actants formels (le segment fonctionnel). Le verbe, au contraire, fait la synthèse des données de l'analyse réalisée par le domaine nominale en les faisant réorganiser et retraiter par le paramétrage en temps, modalité et allocutivité. Cette synthèse est soit réalisée par accrétion pronominale dans le verbe ou l'auxiliaire (conjugaison agglutinante par accord multiple), livrant une forme personnelle, soit suspendue par défaut d'une telle agglutination, auquel cas on obtient une forme impersonnelle munie de repérages aspectuels et compatibles avec diverses fonctions nominales et adjectivales, sans qu'on puisse véritablement parler de forme nominale du verbe à l'échelle de l'unité lexicale. En revanche, une proposition terminée par un verbe (personnel ou impersonnel) ou un auxiliaire (personnel) peut être prise pour segment nominal d'un SN enchâssant, auquel cas son dernier constituant lexical (verbe ou auxiliaire) recevra les postpositions (détermination, nombre, cas) constitutives du segment fonctionnel assignant un rôle syntaxique à l'ensemble propositionnel. Ceci laisse l'impression que les participes sont universellement nominalisables et admettent tous les traitements. Il faut faut de même distinguer le cas d'une forme expressément impersonnelle et déverbale (ikuste) d'une qui ne l'est pas (ikusi) en s'appuyant sur la distinction langue / discours, mais le traitement des actants régis demeure inchangé.

En définitive, parler de «forme nominale du verbe basque »n'a aucun sens si on ne discerne pas dès le départ que la question de la nominalité ne se pose pas dans les mêmes termes à l'échelle de l'unité lexicale de langue dans son segment notionnel propre et à celle du syntagme au niveau de la proposition. C'est ce distingo que ne font pas les langues romanes et germaniques qui explique pourquoi un infinitif ou un gérondif ne peuvent conserver un sujet nominal ou clitique de morphosyntaxe «nominative " égale à celui du sujet du verbe d'une indépendante: je suis parti, *il a vu je partir / partant. La syntaxe basque fonctionne sur le modèle il a vu [je part]-ir, esquivant le reprofilage des connexions verbe-actants par la translation du verbe lexical et les prépositions ou changement de cas. Les langues romanes résolvent le problème en rapportant les clitiques du verbe régi directement au verbe recteur sous la forme de clitiques objet (il m'a vu partir), et les langues celtiques au contraire ont des pronoms objets en réalité possessifs, d'emblée compatibles avec les formes nominales du verbe. Le basque, avec ses nominalisations propositionnelles macrocosmiques sans effet sur les relations microcosmiques entre constituants, n'a aucun problème de cet ordre à résoudre. Le basque est une langue fortement cloisonnée qui tend à affecter la gestion des questions sémantiques à des micro-modules étanches qui contribuent individuellement à la construction du sens d'ensemble en fournissant des éléments qui se cumulent aux autres par composition cumulative sans en passer par des échanges osmotiques dans lesquels l'architecture des systèmes se contamine mutuellement. La relative autonomie des domaines nominal et verbal, la fragmentation des segments notionnel et fonctionnel, la conjugaison périphrastique en sont autant d'exemples. La question des formes nominales du verbe et l'autonomie entre la structure interne et le rôle externe de la proposition est l'exemple par excellence de ce cloisonnement, et il s'inscrit harmonieusement dans les tendances organisationnelles qui caractérisent 
toute l'architecture du basque et la singularisent en regard du reste de l'aire linguistique européenne.

\section{Abréviations}

\section{abréviation Catégorie}

A

ABL

ADL

ALL

B0

B1

B2

$\mathrm{COM}$

CONJ

$\mathrm{CP}$

CS

D

E

GER

HYP

IN

INSTR

INT

GL

GP

NEG

NV

PA

PART

PAS

PI

PP

RCP

REL

RES

SUBJ

SUP

1

$1+$

$2 \mathrm{t}$

$2 \mathrm{v}$ absolutif

ablatif

adlatif

allocutif masc./fém./

base zéro

base un

base deux

comitatif

conjonctif

conjug. périphrastique

conjug. synthétique

datif

ergatif

gérondif

hypothétique

inessif

instrumental

interrogatif

génitif locatif

génitif possessif

négation

nom verbal

participe accompli

partitif

passé

participe inaccompli

participe prospectif

réciproque

relatif

résultatif

subjonctif

superlatif

$1^{\mathrm{e}}$ personne

$1^{\mathrm{e}}$ pers. plurielle

$2^{\mathrm{e}}$ pers. tutoyée

$2^{\mathrm{e}}$ pers. vouvoyée
Marqueurs

$-t i k$

-ra

-ka-/-k/-a-, -na-/-n, h- (neutralisé)

-kus-

ikus

ikusi "voir" (accompli)

-ekin

$-l a$

participe + auxiliaire

Verbe

$-(r) i$

$-k$

-tzen, -zten, -ten

$-k e$

$-n$

$-z$

-en (suffixe verbal)

$-k o$

$-(r) e n$

ez.

-tze-

B2 (ikusi)

$-(r) i k$

$-n$

B0-tzen, -(z)ten (ikusten)

PI-GL (ikusiko)

elkar

$-n$

$-t a,-d a$

$-z a-$

-en

$n-,-d a-,-t$

$\mathrm{N}: g u, \mathrm{~V}:-g u-$

$\mathrm{N}: h i, \mathrm{~V}: k a /-a-/-k$ (masc.), na/-n (fém.)

$\mathrm{N}: z u, \mathrm{~V}:-z u-$ 


$\begin{array}{lll}2 \mathrm{v}+ & 2^{\mathrm{e}} \text { pers. vouv. pl. } & \mathrm{N}: \text { zue, } \mathrm{V}:- \text { zue- } \\ 3 & 3^{\mathrm{e}} \text { pers. } & \mathrm{N}: \text { hura, } \mathrm{V}: \text { allomorphes } \\ 3+ & 3^{\mathrm{e}} \text { pers. pl. } & \mathrm{N}: \text { haiek, } \mathrm{V}: \text { allomorphes }\end{array}$

\section{Références}

Adamczewski H. \& Delmas C., 1982, Grammaire Linguistique de l'Anglais, Colin, Paris.

Anderson S., 1976, On the Notion of Subject in Ergative Languages, Charles N. Li (ed), Subject and Topic, New York, Academic Press, 1-23.

Bottineau D., 2005, Prédication et interaction cognitive en basque, Mémoires de la Société de Linguistique, XIV, Peeters, Louvain, p. 97-132.

Bottineau D., 2006, Les formes non finies du verbe basque, Blanvillain, O. \& Guimier, C., Travaux linguistiques du Cerlico, 19, Les formes non finies du verbe -1-, Presses Universitaires de Rennes. https://hal.ccsd.cnrs.fr/ccsd-00097953

Bottineau D., 2007, Ergativité nominale et actance verbale en basque, Actes $d u$ $11^{e}$ Colloque International de Psychomécanique du Langage, Université Paul Valéry, Montpellier, 8-10 juin 2006.

Bottineau D., 2008a (à paraître), Language and enaction, J. Stewart, O. Gapenne, \& E. Di Paolo (eds), Enaction: towards a new paradigm for cognitive science, MIT.

Bottineau D., 2008b (à paraître), La morphosyntaxe allocutive du sens grammatical », Revue de Sémantique et Pragmatique 19/20, 71-98.

Bottineau D., 2008c (à paraître), Personne de langage et personne de langue en basque, in J. Vicente Lozano et A. Leblanc, Les représentations linguistiques de la personne, Presses Universitaires de Rouen.

Bottineau D. \& Roulland D., 2007a, Le problème de la réflexivité en basque, D. Bottineau, D. Roulland \& A. Rousseau (eds), L'énoncé réfléchi, Presses Universitaires de Rennes, 205-228.

Bottineau D. \& Roulland D., 2007b, La grammaticalisation l'adresse en basque : tutoiement et allocutivité, L. Begioni et C. Muller, (dir.), Problèmes de sémantique et de syntaxe, Hommage à André Rousseau, Editions du Conseil Scientifique de l'Université Charles-de-Gaulle Lille 3, collection UL3 travaux et recherches, 351-372.

Coyos J.-B., 1999, Le parler basque souletin des Arbailles, une approche de l'ergativité, L'Harmattan.

Coyos J.-B., 2002, Parcours de type passif et de type antipassif en basque souletin parlé actuel, Bulletin de la Société de linguistique de Paris, t. XCVII, fasc. I, p. 283-314.

Hualde J. I. \& Ortiz de Urbina J. (eds.), 2003, A Grammar of Basque, Mouton de Gruyter.

Lafitte P., (1944 $\left.{ }^{1}, 1962^{2}\right)$ 1979, Grammaire basque (navarro-labourdin littéraire), Elkar.

Mandelbrot B., 1975, Les objets fractals, Paris, Flammarion.

Pottier B., 1992, Sémantique générale, PUF.

Rebuschi G., 1978, Cas et fonction sujet en basque, Verbum 1.1, 69-98.

Rebuschi G., 1984, Structure de l'énoncé basque, Paris, SELAF. 
Robert S. (éd.), 2003, Perspectives synchroniques sur la grammaticalisation, Peeters, Louvain-Paris.

Rotaetxe K., 1978, Linguística-logica: La construcción ergativa vasca, Revista Espanola de Lingüística Madrid, vol. 8, n 2 , pp. 431-445.

Roulland D., 2002, Gustave Guillaume et la langue basque, in R. Lowe (ed), Le système des parties du discours, sémantique et syntaxe, Laval, Presses de l’Université Laval, p. 383-395.

Roulland D., 2004, Perfectivité et schémas actanciels en basque, Genèse de la "phrase" dans la diversité des langues, Modèles Linguistiques, tome XXV, fascicules $1 \& 2$, p. 305-320.

Toussaint M., 2003, Analogiques, P. Monneret, Cahiers de linguistique analogique 1, Le mot comme signe et comme image: lieux et enjeux de l'iconicité linguistique, ABELL, Dijon.

Trask R. L., 1981, Basque verbal morphology, in IKER 1, Rencontres internationales de bascologues, Académie de la langue basque, Bilbao. 